\title{
EL PROBLEMA DEL
}

\section{THE PROBLEM OF}

\section{CRECIMIENTO SOSTENIBLE ¿CÓMO CRECER SIN} DESTRUIRSE?

\section{SUSTAINABLE GROWTH- HOW TO GROW WITHOUT SELF-DESTRUCTION?}

FECHA DE RECEPCIÓN: 8 de mayo FECHA DE APROBACIÓN: 29 de julio Pp. 136-147

\author{
Jorge Saravia Rios*
}

* Ingeniero de Sistemas, Escuela Colombiana de Ingeniería, (Bogotá, Colombia 1988). Magíster en Administración Universidad de los Andes (Bogotá, Colombia 1991). Profesor de planta Universidad de los Andes Facultad de Administración (1993-2004); Profesor de cátedra Universidad de los Andes, Facultades de Administración y Derecho (2004

a la fecha). Consultor en temas de estrategia financiera, creación de valor y sostenibilidad corporativa (2004 a la fecha). Miembro Principal de la Junta Directiva de Protecsa S.A. Publicación del artículo Complejidad, equilibrio, $y$ finanzas: conceptos y herramientas para la gestión de empresas sostenibles.

Publicaciones en curso sobre gerencia de valor, planeación financiera y teoría de costos, y estrategia. Participación con la Facultad de Administración de la Universidad de los Andes en los proyectos Expopyme y RDI. 


\section{RESUMEN}

De una u otra forma las organizaciones perciben el crecer como una meta necesaria en la planeación estratégica y cuando se logra el crecimiento buscado se supone una gestión exitosa. Sin embargo, en ocasiones no hay claridad sobre lo que significa crecer, cuáles son sus efectos y no se comprende con profundidad el significado de una buena gestión.

Es común ver a las organizaciones formular preguntas como:¿Crecer significa vender más?, ¿Hasta dónde puedo crecer? ¿Crecer es tener mayor infraestructura y personal? ¿Qué tan rentable seré creciendo? o presentando afirmaciones como: "la meta del plan estratégico para este año es crecer un 20\%, eso nos posicionará como el número uno en el sector". La meta de una organización debe ser: ser sostenible y el crecer, puede ser una estrategia para lograrla, sin embargo, el crecer por sí mismo no necesariamente genera sostenibilidad, por esto, si una organización lo entiende como su meta puede comprometer su viabilidad. El problema del crecimiento tiene varias dimensiones de análisis que involucran todas las instancias de una organización, por ejemplo, se necesita tener acceso a la información del mercado y el entorno, se necesita tener la inteligencia para comprender esta información, se necesita una estructura organizacional dinámica, flexible e innovadora, es necesario comprender cómo los diferentes procesos interactúan y qué recursos necesitan. Para entender el crecer como una estrategia, se hace necesaria su formalización, y el uso de herramientas que permitan su manejo, estas herramientas deberán incluir las diferentes dimensiones de análisis. Mediante el uso de estos modelos, una organización puede diseñar una estrategia de crecimiento coherente con sus metas, $y$ sin el riesgo de comprometer su estabilidad.

\section{ABSTRACT}

In one way or another, organizations perceive growth as a necessary goal in strategic planning, and when it is reached as expected, it becomes a success. However, in certain occasions, it is not clear what growing means, what its effects are, and there is no understanding of the meaning of an effective management model.

It is common to see organizations asking themselves questions like: does growing mean selling more?, to what extent can I grow?, does growing mean having a bigger infraestructure and personnel?, how much profitable would I be growing?; or having affirmations like: the goal of a strategic plan for this year is to grow $20 \%$, this will position us as number one in this sector. The goal of an organization will be: to be sustainable, and to grow; however, growing itself doesn't necessarily generate sustainability, for this, if an organization takes it as its goal, it could affect its feasibility. The problem of growing has several domensions of analysis which involve all parts of an organization. For example, an organization needs to have access to information on the market and return, it needs to understand this information, and it needs a dynamic organizational structure, flexible and innovative, it also needs to understand how different processes interact and which resources are needed. To understand growing as a strategy, it is necessary its formalization and the use of tools that allow its management; these tools should include several analysis dimensions. Through the use of these models, an organization could design a growing strategy according to its goals, and without the risk of affecting its stability.

\section{RESUMÉÉ}

D'une manière ou d'une autre, les organisations perçoivent leur croissance comme un objectif nécessaire dans la planification stratégique. Quand le taux de croissance recherché est obtenu, cela suppose qu'une gestion adéquate a été réalisée. Cependant, il n'y a parfois pas de clarté suffisante sur ce que le concept de croissance signifie, quels en sont ses effets, et la signification d'une bonne gestion n'est pas comprise en profondeur.

Il est commun de voir des organisations formuler des questions du type : Crôttre signifie t-il vendre plus ? Jusqu' où pouvons-nous crôtre ? Crô̂tre est signifie avoir une plus grande infrastructure et plus de personnel ? Serions nous plus rentable si notre croissance s'améliorerait ? ou de prétendre à des affirmations du type : l'objectif du plan stratégique de cette année est une croissance de 20\%, cela fera de nous les numéro un du secteur. L'objectif d'une organisation doit être : être viable, produire de la croissance grâce à une stratégie efficace. Cependant, n'avoir que la croissance pour objectif ne produit pas nécessairement une aptitude à soutenir cette croissance de manière prolongée, pour cela, si une organisation en fait son objectif fondamental, elle peut compromettre sa propre viabilité. Le problème de la croissance a plusieurs dimensions d'analyse intégrant tous les départements d'une organisation, par exemple, il faut avoir accès à l'information du marché et à son environnement, il faut faire preuve d'intelligence pour comprendre cette information, on a besoin d'une structure organisationnelle dynamique, flexible et innovatrice, il est nécessaire de comprendre comment les différents processus interagissent et de quelles ressources il faudra tenir compte. Pour comprendre la croissance comme une stratégie, sa concrétisation devient nécessaire, et l'utilisation d'outils qui permettent son maniement devra inclure les différentes dimensions de l'analyse. Par une utilisation de ces modèles, une organisation peut concevoir une stratégie de croissance cohérente avec ses objectifs, sans risque de compromettre sa stabilité.

\section{Palabras claves}

\author{
Crecimiento \\ Sostenibilidad \\ Sostenible \\ Tasa \\ Creación de valor \\ Finanzas corporativas \\ Gestión \\ Estrategia \\ Contabilidad.
}

\section{Key words}

$$
\begin{gathered}
\text { Growing } \\
\text { Sustainability } \\
\text { Sutainable } \\
\text { Rate }
\end{gathered}
$$

Value creation

Corporative finances

Management

Strategy, accountability 


\section{INTRODUCCIÓN}

S frecuente encontrar empresas que luego de cumplir con metas estratégicas en términos de crecimiento tales como "lograr una participación del 10\% del mercado", enfrentan serios problemas de sostenibilidad. Esta aparente contradicción obliga a preguntarse sobre el por qué el cumplimiento de una meta expresada en términos de crecimiento puede generar un deterioro en la estructura de una empresa, que inclusive puede llevarla a su disolución, cuáles son las causas de este comportamiento y cuáles son las herramientas que permiten al empresario abordar el tema del crecimiento sin que con esto comprometa el futuro de su empresa. Esta reflexión hace también necesario cuestionar el hecho de si se debe o no considerar el crecimiento como una meta, o si este simplemente debe ser entendido como un objetivo o una estrategia, que en las condiciones adecuadas puede consolidar la estructura de

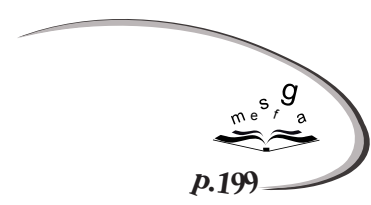
una empresa maximizando su potencial para ser sostenible.

Pero el problema no se limita al cuestionar si el crecer debe ser un fin o un medio, también es importante entender y formalizar qué significa crecer, entender cuáles son las estructuras que afecta y cuál es la relación del crecimiento con el estado de sostenibilidad de una empresa.

Si bien pueden existir diferentes ópticas para abordar la problemática descrita, las finanzas ofrecen modelos que permiten una comprensión clara y objetiva del tema del crecimiento, entender la relación con las diferentes estructuras de una empresa y comprender la relación entre el crecimiento y la sostenibilidad.

El presente artículo expondrá el tema del crecimiento desde su definición, se presentarán los problemas que puede enfrentar una empresa, derivados de entender el crecimiento como una meta en sí misma y/o crecer inadecuadamente, y se presentarán herramientas que sirven como base para desarrollar una estrategia de crecimiento alineada con una meta de sostenibilidad. 


\section{2. ¿QUÉ SIGNIFICA CREEER?}

La primera asociación que se puede hacer cuando se habla de crecimiento en una empresa es su relación con el tema de ventas. Es común entender el crecimiento en términos de la variación periódica positiva de la cifra de ventas, de esta forma el crecimiento se expresa como:

En donde

$$
\Delta \% \text { Ventas }>0
$$

$$
\Delta \% \operatorname{Ventas}=\left(\operatorname{Ventas}_{\mathrm{i}}-\operatorname{Ventas}_{(\mathrm{i}-1)}\right) /\left(\operatorname{Ventas}_{(\mathrm{i}-1)}\right)
$$

En donde i se refiere a un período específico.

Las ventas de una empresa, ya sea de productos tangibles, comercializadoras o industriales, o de productos intangibles, de servicios, siempre tienen dos componentes:

$\Rightarrow$ Cantidad vendida en términos de su unidad de facturación.

\section{$\Rightarrow$ Precio.}

Por lo tanto el crecimiento en ventas se descompone así:

$$
\Delta \% \text { Ventas }=((1+\Delta \% \text { Cantidad }) *(1+\Delta \% \text { Precio }))-1
$$

En donde:

$\Delta \%$ Cantidad $=\left(\right.$ Cantidad $_{\mathrm{i}}-$ Cantidad $\left._{(\mathrm{i}-1)}\right) /\left(\right.$ Cantidad $\left._{(\mathrm{i}-1)}\right)$ Y

$$
\Delta \% \text { Precio }=\left(\text { Precio }_{\mathrm{i}}-\text { Precio }_{(\mathrm{i}-1)}\right) /\left(\text { Precio }_{(\mathrm{i}-1)}\right)
$$

En donde i se refiere a un período específico.

La segunda asociación que se puede hacer con referencia al tema del crecimiento, es su relación con la infraestructura, que en una empresa está representada por su activo, pero es necesario entender que la infraestructura no se refiere únicamente al activo fijo, también incluye capital de trabajo y otros activos. Esta segunda forma de crecimiento se define en términos de una variación positiva en el activo y se expresa como:

En donde:

$$
\Delta \% \text { Activo }>0
$$

$$
\Delta \% \operatorname{Activo}=\left(\operatorname{Activo}_{\mathrm{i}}-\operatorname{Activo}_{(\mathrm{i}-1)}\right) /\left(\operatorname{Activo}_{(\mathrm{i}-1)}\right)
$$

En donde i se refiere a un período específico.

Antes de analizar la relación entre las dos formas de crecimiento y la sostenibilidad, es necesario entender con mayor profundidad cada uno de estos conceptos, sus conexiones y su relación con la estructura de la empresa.
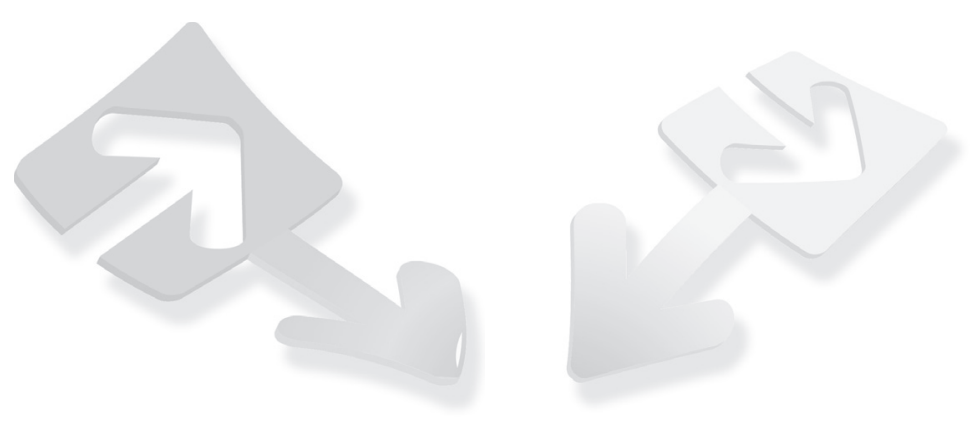


\section{EL CRECIMIENTO EN VENTAS}

Como se mencionó, el crecimiento en ventas involucra las variaciones en cantidad y precio, de estas dos variables quizás la que demanda mayor atención es el precio. Cuando se pregunta al responsable del manejo de precios en una empresa por su definición, es común recibir una respuesta en términos de que el precio resulta de agregar un margen al costo unitario del producto o servicio, es decir que precio se definiría como:

Precio $=$ Costo unitario de producción $*(1+$ Margen $\%)$

En donde el costo unitario de producción sería el resultante de establecer el total de recursos consumidos por una unidad producida, de acuerdo con una metodología de costeo usada; el Margen sería el porcentaje que se busca percibir por encima del costo, esto es equivalente a suponer que el costo se recupera con el precio. Es importante anotar que el término costo de producción incluye a las empresas de prestación de servicios, este tipo de empresa se comporta como una empresa industrial que trabaja por pedido, pero con una cadena de producción más difícil de definir ya que el producto es intangible.

Bajo esta óptica, un crecimiento en ventas se podría lograr solamente con una variación positiva en precios, sin tener que crecer en volumen las unidades vendidas, lo que representa desde la estructura del estado de resultados de una empresa el menor esfuerzo posible ya que, asumiendo la cantidad constante, no habría necesidad de un incremento en costos y gastos para generar el crecimiento buscado, esto significa que:

\section{$\Delta \%$ Ventas $=\Delta \%$ Precio}

Este supuesto puede ser válido en condiciones específicas pero no es la generalidad. Lo anterior se cumple solamente si la cantidad vendida es una variable independiente del precio, esto quiere decir que el producto que la empresa vende no tiene sustitutos, no hay competencia, se está en un nicho de mercado, es decir, la empresa es o se comporta como un monopolio; sin embargo, con la tendencia global a la no diferenciación de los productos, es prudente suponer a las empresas como insertas en algún tipo de mercado competitivo quizás aproximándose a una competencia perfecta y no a competencia imperfecta (Samuelson: Nordhaus, 1986), en la cual la variable cantidad vendida es dependiente de la variable precio. La relación que pueda existir entre la demanda y el precio se define como: elasticidad de la demanda, que indica el grado de respuesta de la cantidad demandada a las variaciones del precio de mercado, esta relación se puede expresar en su forma básica como:

\section{Elasticidad $=\Delta \%$ Cantidad $/ \Delta \%$ Precio}

Cuando esta relación es menor que - 1 tenemos una demanda elástica, cuando la relación es igual a-1 tenemos una elasticidad unitaria, cuando la relación de la cantidad en muy pequeña frente a la variación del precio tenemos una demanda inelástica (Samuelson- Nordhaus, 1986). En general tenemos que:

\section{$\Delta \%$ Cantidad $=-$ Elasticidad $* \Delta \%$ Precio}

Si se analiza con cuidado la expresión anterior, es fácil concluir que cuando una empresa tiene una demanda elástica y se busca un crecimiento en ventas, no se puede pensar en el precio como un mayor valor del costo, en su lugar se debe suponer un precio fijado por el mercado y si se busca crecer en ventas, se debe buscar un precio que permita el crecimiento buscado en cantidad, es decir, el precio se forma con la siguiente expresión:

\section{Precio futuro $=$ Precio actual $*(1-(\Delta \%$ Cantidad $/$ Elasticidad))}

En donde la $\Delta \%$ Cantidad se refiere al porcentaje en el que la empresa buscaría incrementar su cantidad vendida. 


\section{EL CRECIMIENTO EN ACTINOS}

El aumento en cantidad vendida puede generar exigencias en la estructura de costos y en la de gastos de ventas de la empresa, el efecto conjunto de estos factores es un cambio en margen de utilidad, que se refiere a la comparación entre la utilidad neta y las ventas. Esta estructura puede generar otro requerimiento y es el referente a la capacidad instalada, si esta no es suficiente para el volumen de ventas buscado, la empresa incurrirá en un aumento necesario en el activo fijo, el cálculo de este aumento se puede expresar como:

$\%$ Activo Fijo productivo $=\mathbf{F}(\Delta \%$ Cantidad $)$

En donde $\mathrm{F}$ es la función que relaciona la variación en volumen adicional en cantidad a vender con el requerimiento adicional de activo fijo productivo, esta función F debe ser calculada para cada empresa, incluyendo las empresas de prestación de servicios.
Además del requerimiento en activo fijo, existe otra variación en el activo que es menos clara pero que es inevitable, y es el crecimiento en el activo corriente. Si las ventas crecen, una consecuencia directa es el aumento en caja y cartera, también puede generarse una variación en inventarios si la empresa los maneja, esta variación se puede expresar como:

$\%$ Activo corriente $=\Delta \%$ Caja $+\Delta \%$ Cartera + $\Delta \%$ Inventarios

Como consecuencia, el crecimiento total en activos derivado de un crecimiento en ventas, se puede expresar como:

$\Delta \%$ Activos $=\Delta \%$ Activo Fijo productivo $+\Delta \%$ Activo corriente
Se han presentado dos formas mediante las cuales una empresa puede crecer y se ha mostrado que existe una relación entre el crecimiento en ventas y el crecimiento en activos, hasta el momento se ha presentado el crecimiento como una actividad natural de un negocio y se dejó claro

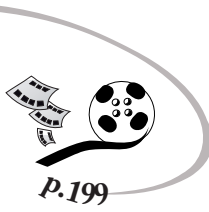
que teniendo una comprensión del mercado en el cual la empresa se mueve y dimensionando correctamente los requerimientos de infraestructura se pueden lograr los objetivos de crecimiento. Sin embargo hay una dimensión que no es tenida en cuenta cuando el crecimiento se aborda desde una óptica únicamente comercial, de mercadeo y operativa.

Si se retoman las definiciones básicas del modelo contable encontramos que un estado de resultados habla de una gestión de entrega de productos y de consumo de recursos, todo basado en el principio de causación. Por lo tanto, el efecto de un crecimiento en ventas se refleja como un crecimiento en la utilidad que formará parte del patrimonio de la empresa, teniendo en cuenta que se pueden repartir utilidades, por otro lado, asociado con este crecimiento en ventas, hay un crecimiento en activos y como consecuencia de la estructura contable, este crecimiento en activos debe ser financiado por la estructura de pasivos y patrimonio. Si bien el patrimonio financia parte del crecimiento en activos con la retención de las utilidades, esto puede no ser suficiente, es por eso que el pasivo también necesitaría crecer para financiar el resto del crecimiento del activo. Si una empresa aumenta su pasivo para financiar su crecimiento en activo, pero mantiene su endeudamiento, porcentaje del activo financiado con pasivos, podrá mantener un 
equilibrio en su estructura, pero si el crecimiento en activo genera un aumento en el endeudamiento, se puede detonar una situación que exigirá recursos costosos para financiar activo que puede ser no productivo.

El problema del crecimiento se da porque crecer cuesta, requiere financiación y cuando la necesidad de financiación del crecimiento con pasivos supera la capacidad de financiación interna de la empresa, esto es retención de utilidades y rompe el equilibrio de la estructura de capital, se genera una inestabilidad que de mantenerse compromete la sostenibilidad de una empresa.

Cuando el crecimiento se comprende únicamente desde la perspectiva de aumentar una participación en un mercado y/o el aumento en infraestructura, una empresa puede fijar metas en función de $\Delta \%$ Ventas y/o $\Delta \%$ Activos, y hacer lo necesario para cumplirlas. Sin embargo, se está ignorando el esfuerzo que la estructura de capital debe hacer para financiar esta meta, por esto antes de crecer, es necesario comprender los límites que la estructura de la empresa impone.

Figura 1. Flujograma de crecimiento de una empresa basado en sus activos corrientes y volúmen de ventas

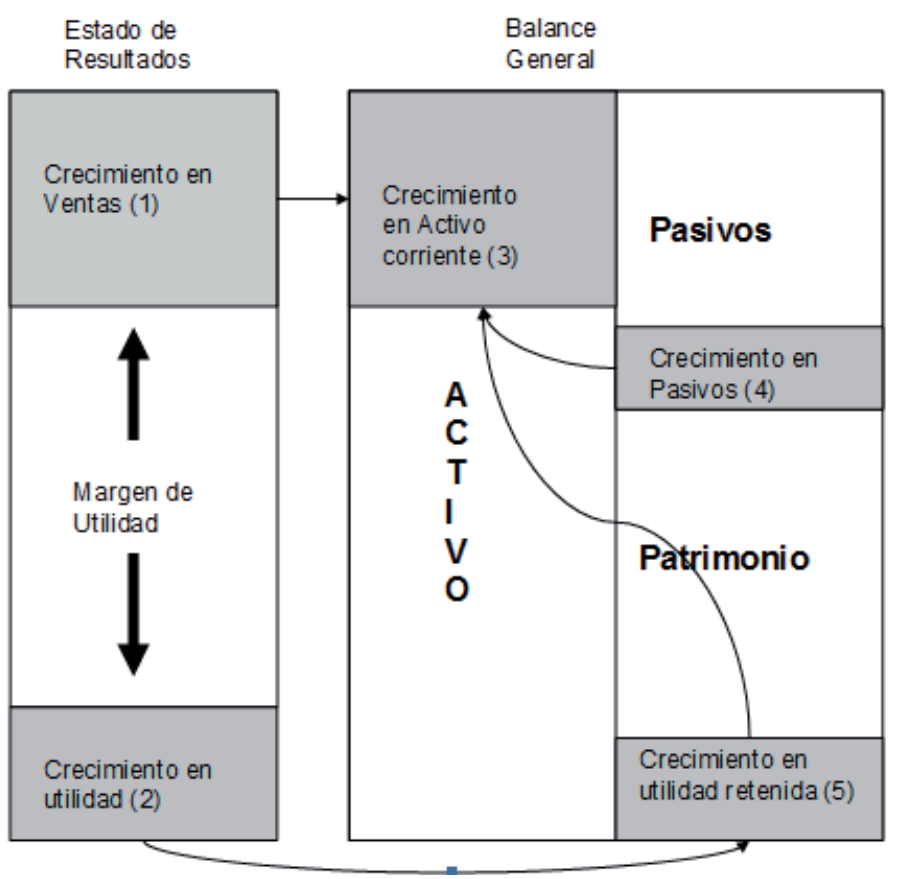

La figura 1 resume las relaciones descritas entre el crecimiento en ventas, en activo y el necesario en la estructura de capital. Para que el crecimiento no comprometa la estabilidad de la empresa, partiendo de un balance que cumpla con la condición Activo=Pasivo + Patrimonio, se deben cumplir dos condiciones:

$$
\begin{gathered}
(3)=(4)+(5) \\
Y
\end{gathered}
$$

Pasivo $\left._{(\mathrm{i}-1)} / \operatorname{Activo}_{(\mathrm{i}-1)}=\left(\operatorname{Pasivo}_{(\mathrm{i}-1)}+\right\}(4)\right) /\left(\operatorname{Activo}_{(\mathrm{i}-1)}+(3)\right)$

en donde:

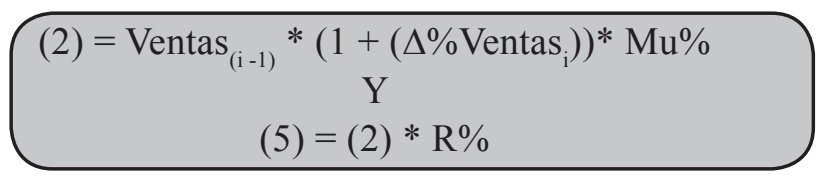

Suponiendo $\mathrm{R} \%$ se refiere al porcentaje de retención de utilidades, $\mathrm{Mu} \%$ en margen de utilidad del estado de resultados, e $\mathrm{i}$ al periodo actual.

Si además del crecimiento en activo corriente la empresa requiere en capacidad instalada, mediante el aumento en el activo a largo plazo, tenemos la situación presentada en la figura 2 . 
Figura 2. Flujograma de crecimiento de una empresa basado en activos a largo plazo

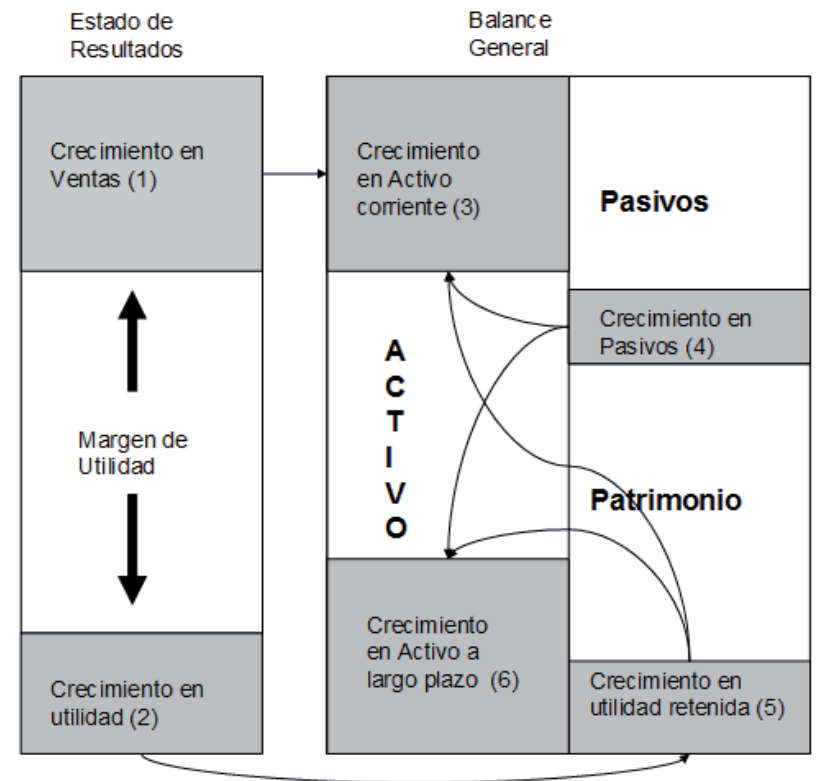

En este caso la presión sobre la estructura de capital para financiar el crecimiento es mayor y las condiciones a cumplir para no comprometer el equilibrio de la empresa se transforman en:

Y

$$
(3)+(6)=(4)+(5)
$$

$$
\begin{gathered}
\text { Pasivo }_{(\mathrm{i}-1)} / \operatorname{Activo}_{(\mathrm{i}-1)}= \\
\left(\operatorname{Pasivo}_{(\mathrm{i}-1)}+(4)\right) /\left(\operatorname{Activo}_{(\mathrm{i}-1)}+(3)+(6)\right)
\end{gathered}
$$

Es necesario tener en cuenta que la única fuente de financiación para el crecimiento en activo a largo plazo no es el pasivo más retención de utilidades. También se puede acudir al capital pagado o aportes, y no se debe ignorar que este tiene un costo. Cuando en lugar de usar el pasivo más la retención de utilidades para financiar el activo se usa el capital, la empresa cambia.

\section{LA TASA DE GREGIMIENTO SOSTENIBLE}

La situación descrita en las figuras 1 y 2 obliga a replantear el tema del crecimiento, la pregunta que una empresa debe hacerse no es ¿Hasta dónde se quiere crecer?, ni ¿Cuál sería la participación de mercado objetivo?, la pregunta a formular es ¿Hasta dónde me permite crecer la estructura de mi empresa?

Del análisis de las relaciones descritas en párrafos anteriores entre crecimiento y los requerimientos de financiación surge la tasa de crecimiento sostenible (TCS) (Ross, et al., 2001), que se define como "la tasa máxima de crecimiento que puede lograr una empresa, sin recurrir a financiamiento externo, por medio de instrumentos de capital, mientras mantiene una razón constante de deuda a capital contable", (Ross, et al., 2001). Este análisis permite dimensionar los límites de crecimiento en ventas de una empresa y presenta un instrumento para su gestión. La TCS se puede expresar en forma general como:

$$
\mathrm{TCS}=\mathrm{ROE} * \mathrm{R} \% /(1-(\mathrm{ROE} * \mathrm{R} \%))
$$

En donde ROE es la razón utilidad neta patrimonio, y $\mathrm{R} \%$ es la tasa de retención de utilidades. 
Este indicador deja ver que el potencial de crecimiento de una empresa es proporcional a su eficiencia operativa en el uso de los recursos patrimoniales e inversamente proporcional a la repartición de utilidades, esto quiere decir que el máximo crecimiento sostenible en ventas se logra con la máxima eficiencia en costos y gastos para un nivel patrimonial y la no repartición de utilidades. Es importante anotar que esta TCS debe construirse para cada empresa, teniendo en cuenta sus propias características, el comportamiento de sus activos y otras particularidades, sin embargo, la fórmula presentada puede usarse como un indicador genérico teniendo en cuenta el supuesto de que todo el activo crece en la misma proporción que las ventas y de que no habrá aumento de capital pagado o aportes.

Figura 3. La tasa de crecimiento sostenible

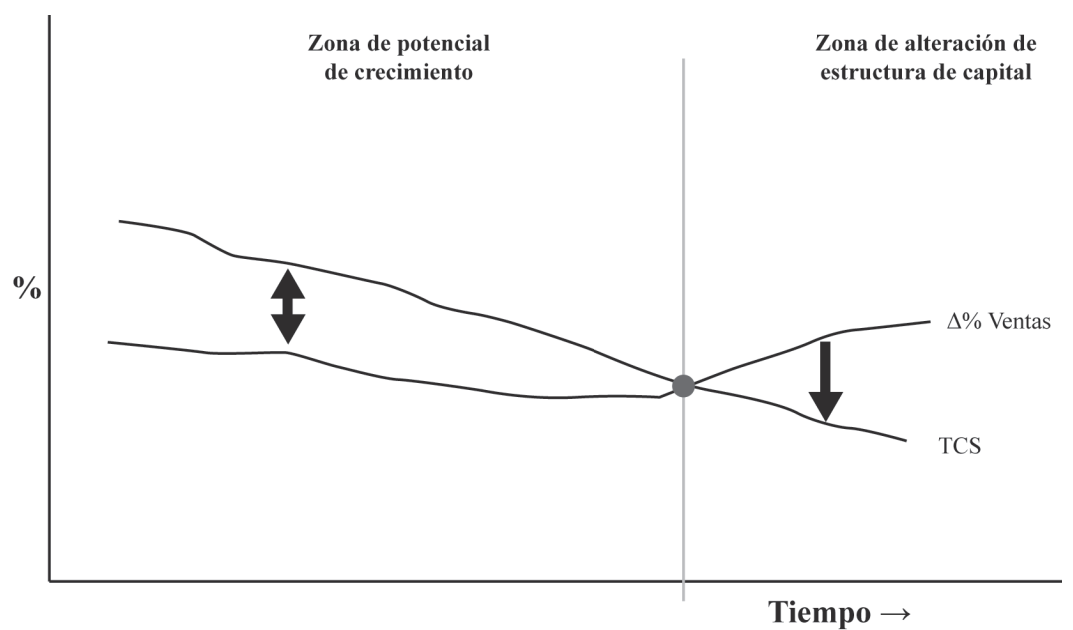

La figura 3 muestra un ejemplo que compara una TCS proyectada y un presupuesto de crecimiento en ventas. En este caso el presupuesto de crecimiento en ventas no guarda relación con la TCS y de no vigilarse el límite calculado, se generaría inestabilidad en la estructura de capital de la empresa.
La importancia de la TCS está en que entrega a la dirección de la compañía un instrumento que pone límites a la gestión comercial, obliga a un trabajo en equipo en el proceso de planeación y obliga a fijar una meta más allá del crecimiento y en términos de equilibrio y sostenibilidad.

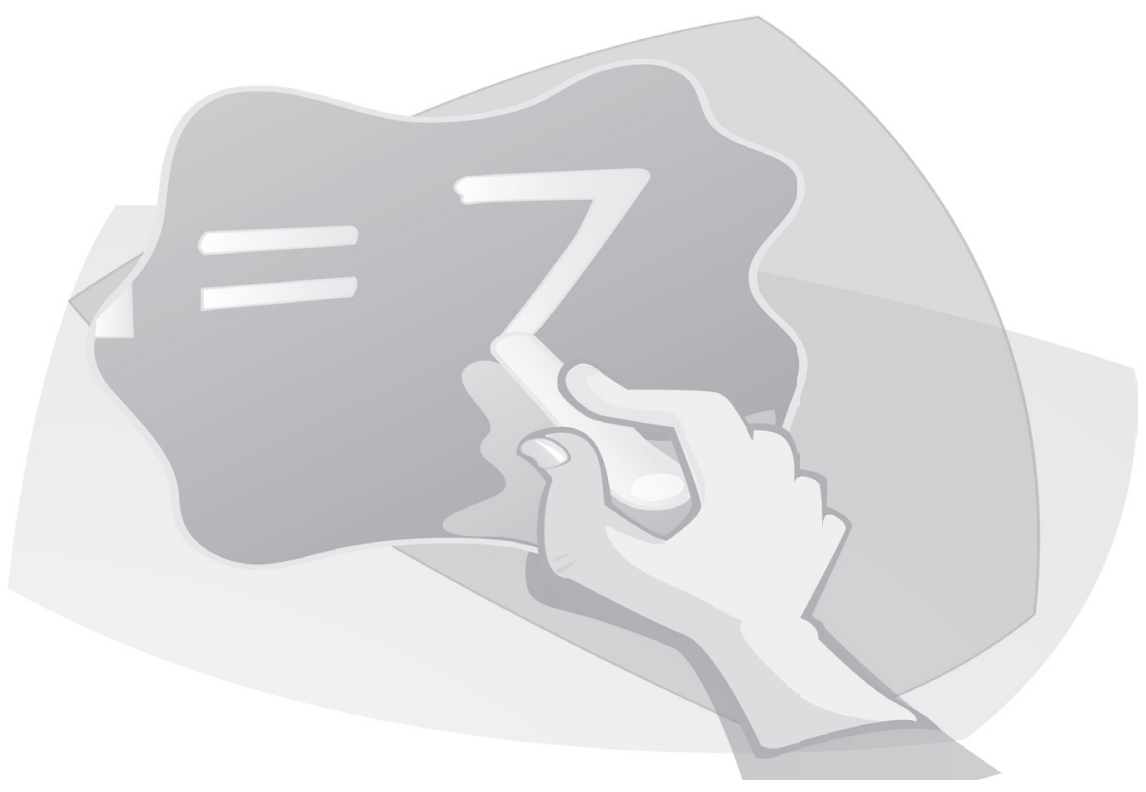




\section{EL GRECIMIENTO Y LA SOSTENIBILIDAD}

Desde la óptica financiera, una empresa sostenible es aquella capaz de tener una generación operacional superior al costo de los recursos que financian su infraes-tructura y mantener esta condición en el tiempo. Uno de los indicadores que permite este análisis es "Valor Económico Agregado (Fernández, 1999) (EVA), que se expresa de la siguiente forma:

$$
\text { EVA }=(\text { ROI - WACC }) \text { * Inversión. }
$$

En donde:

\section{ROI:}

Utilidad operacional * $(1-$ impuestos\%) / Inversión, se refiere a la rentabilidad operacional después de impuestos de los recursos costosos invertidos en infraestructura.

\section{WACC:}

Del inglés Weighted Average Cost of Capital, es el costo promedio ponderado de capital, se refiere al costo promedio que la empresa paga por cada peso conseguido para financiar su infraestructura que equivale a los recursos invertidos).

\section{Inversión:}

Deuda financiera y patrimonio, equivale a los recursos costosos invertidos en infraestructura.

Un análisis básico del EVA permite entender que cuando la inversión crece pero no genera operación en proporción, el ROI disminuye pero el WACC no, deteriorando la generación de valor agregado, disminuyendo la capacidad de la empresa de atender con su operación el costo promedio de sus recursos y como consecuencia se genera una destrucción de valor patrimonial.

Si retomamos el concepto de tasa de crecimiento sostenible, se puede inferir que un crecimiento superior al límite que la estructura de la empresa impone, puede generar un deterioro en el ROI, ya que se genera la necesidad de recursos costosos para financiar el consecuente crecimiento en activo, pero no para generar más operación, teniendo como consecuencia un variación negativa en el EVA, el consecuente deterioro en el valor patrimonial y el compromiso de la sostenibilidad de la empresa.

\section{EL CRECIMIENTO ¿UNA META?}

Una meta debe servir para definir el resultado esperado de un plan, sirve como un punto de referencia para entender en dónde se está desviando el camino, debe ser universal, integradora y con base en esta, se definen objetivos parciales y los responsables. En términos de una empresa, la meta más universal es el ser sostenible. Desde la óptica financiera integra tres estructuras básicas: la operación, la infraestructura y la estructura de capital. La sostenibilidad parte de entender que la empresa necesita recursos costosos para invertir en infraestructura que sea soporte de la operación y que este debe ser capaz de cubrir el costo de los recursos.
Entender el crecimiento como meta, excluye del análisis al esfuerzo de la empresa por financiar lo necesario para soportarlo y no asegura que lo generado operacionalmente pueda cubrir el costo de los recursos. Por consiguiente, no es garantía de maximización del valor patrimonial y no es garantía de sostenibilidad.

Si por el contrario, la meta de una empresa se expresa en términos de un crecimiento en el valor patrimonial, se puede concluir que un objetivo parcial puede ser 
un porcentaje de crecimiento en ventas alineado con una tasa de crecimiento sostenible y derivado de la meta principal, esta situación se puede extender a un crecimiento en activos, generando una estrategia al servicio de la sostenibilidad, pero también se pude concluir que un objetivo puede ser, el no crecer.

\section{4 \\ 9. CONCLUSIONES}

En un entorno complejo en donde la capacidad de una empresa para moverse al ritmo del mercado es una condición de supervivencia, se enfrenta el dilema de salir del mercado o crecer. En ocasiones se prefiere hacer todo el esfuerzo necesario para lograr la participación buscada, para no perder un cliente, o para ganar un contrato, elevando al nivel de meta la necesidad de estar en el mercado. Sin embargo, esta es una visión parcial del problema ya que en ningún momento la empresa está preguntándose si tiene la agilidad necesaria para mover su estructura y atender los requerimientos que la velocidad del mercado impone y cuál es el efecto real de estos movimientos. A esto se suma el hecho de que el incentivo en muchas organizaciones es proporcional a las ventas y no a la capacidad de hacer gestión sostenible.

¿Cuál debería ser entonces la prioridad? ¿Cómo se toma la decisión de crecer? En los párrafos anteriores se ha mostrado que antes que crecer es necesario comprenderse como una máquina de transformación de recursos, entender que estos recursos cuestan y que la gestión tiene sentido, si lo generado supera los costos asociados a los recursos.

Esta estructura supone una meta que es la maximización del valor patrimonial, así la primera pregunta que se debe resolver es ¿Cuál es la meta en términos de sostenibilidad? Esto es ¿En cuánto se busca maximizar el valor patrimonial?

En este punto modelos como la medición del valor patrimonial a partir del EVA, permiten establecer los modelos de operación, infraestructura y estructura de capital adecuados. Luego, a partir de lo establecido vendría la segunda pregunta, ¿Cuál es el mercado con las condiciones para ser sostenible?

Es aquí en donde se debe reflexionar hacia dónde dirigir el esfuerzo, es necesario conocer su estructura, el comportamiento de la elasticidad de la demanda, es necesario comprender al cliente, el producto y todos los factores que serán la base para el crecimiento en ventas. En este momento surge la necesidad de responder una tercera pregunta ¿Cuánto cuesta entrar en ese mercado? Esto implica cuantificar los recursos necesarios para procesos de innovación y cambio organizacional necesarios. Luego de este análisis surge una cuarta pregunta. ¿Cuáles son los límites que la estructura impone al crecimiento?

La TCS da una respuesta y define dos estructuras sobre las que se pueden actuar, el ROE y la retención de utilidades. Luego viene la quinta pregunta $i$ Tengo la capacidad de producción necesaria? Si bien la TCS presentada en párrafos anteriores parte de una capacidad de producción suficiente, el análisis de este punto puede generar la necesidad de una mayor infraestructura, cuya inclusión en la empresa exige el recálculo de los límites de crecimiento.

\section{Figura 4. El crecimiento sostenible}

\section{EL CRECIMIENTO SOSTENIBLE}

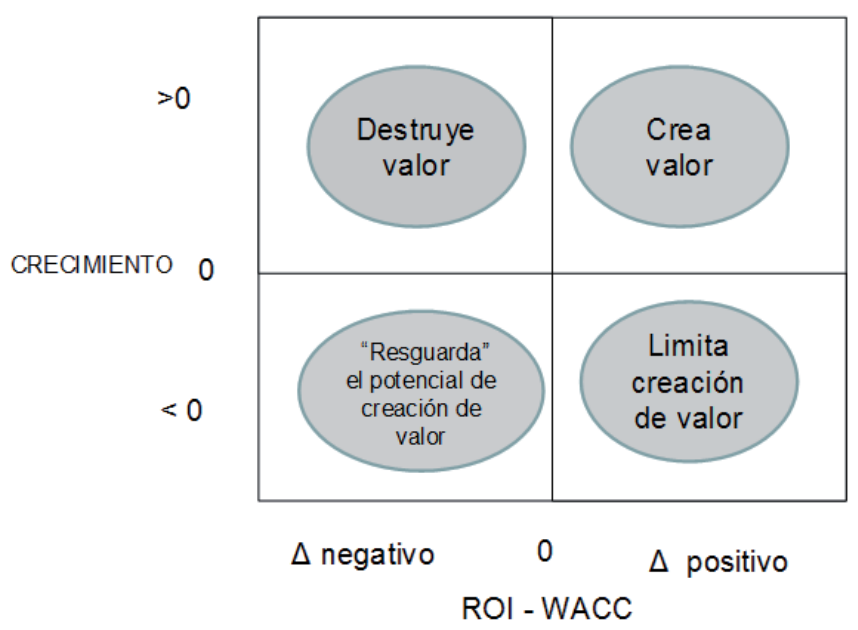


Teniendo en cuenta que la relación ROI-WACC de una empresa determina su capacidad de ser sostenible y teniendo claro que la meta de una empresa debe ser el ser sostenible, es posible establecer los estados que favorecen el crecimiento y aquellos en que la empresa no debería crecer, en la figura 4 es posible observar que no siempre crecer es una opción estratégica válida.
Mediante el uso de la metodología planteada se puede crecer en forma armónica, sin comprometer la sostenibilidad y sobre todo entendiendo que el crecimiento es solo un medio para alcanzar la meta de la organización, que permite fijar objetivos puntuales, pero que no puede suplantar la necesidad de maximizar la capacidad de supervivencia de una empresa, entendida esta como la maximización de valor patrimonial.

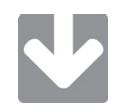

\section{BIBLOGRAFí́}

Fernández, P. (2007). Valuing companies by cash flow discounting: ten methods and nine theories. IESE Business School, University of Navarra.

Fernández, P. (1999). Valoración de empresas, Gestión 2000.

Bennett, G. (2000). En busca del valor, Gestión 2000.

Ross I. et al., (2003). The EVA Challenge: Implementing Value-Added Change in an Organization, Wiley, John \& Sons, Inc.

Ross, et al., (2001). Fundamentos de Finanzas Corporativas, Quinta edición, Mc Graw Hill.

Samuelson y Nordhaus (1986). Economía, Mc Graw Hill. 